\title{
Non-Linear Swing-Up and Stabilizing Control of an Inverted Pendulum System
}

\author{
Marvin Bugeja \\ Faculty of Engineering University of Malta \\ Msida (MSD06) \\ Malta \\ email:merv@ieee.org
}

\begin{abstract}
This paper presents the design and implementation of a complete control system for the swing-up and stabilizing control of an inverted pendulum. In particular, this work outlines the effectiveness of a particular swing-up method, based on feedback linearization and energy considerations. The power of modern state-space techniques for the analysis and control of Multiple Input Multiple Output (MIMO) systems is also investigated and a sfate-feedback controller is employed for stabilizing the pendulum. Cascade control is then utilized to reduce the complexity of the complete controller by splitting it into two separate control loops operating at well distinet bandwidths.
\end{abstract}

Index Terms- Cascade control, Feedback linearization Inverted pendulum, State-feedback control.

\section{INTRODUCTION}

$\mathrm{B}$ EING an under-actuated mechanical system and inherently open loop unstable with highly non-linear dynamics, the inverted pendulum system is a perfect test-bed for the design of a wide range of classical and contemporary control techniques. Its applications range widely from robotics to space rocket guidance systems. Originally, these systems were used to illustrate ideas in linear control theory such as the control of linear unstabie systems. Their inherent non-linear nature helped them to maintain their usefulness along the years and they are now used to illustrate several ideas emerging in the field of modern non-linear control.

A Single rod Inverted Pendulum (SIP) consists of a freely pivoted rod, mounted on a motor driven cart. With the rod exactly centred above the motionless cart, there are no sidelong resultant forces on the rod and it remains balanced as shown in Figure 1.la. In principle it can stay this way indefinitely, but in practice it never does. Any disturbance that shifts the rod away from equilibrium, gives rise to forces that push the rod farther from this equilibrium point, implying that the upright equilibrium point is inherently unstable as shown in Figure 1.1b. Under no external forces, the rod would always come to rest in the downward equilibrium point, hanging down as shown in Figure 1.1c. This is called the pendant position. This equilibrium point is stable as opposed to the upright equilibrium point.
The control task is to swing up the pendulum from its natural pendant position and to stabilize it in the inverted position, once it reaches the upright equilibrium point. The cart must also be homed to a reference position on the rail. All this is achieved only by moving the cart back and forth within the limited cart travel along the rail.

The inverted pendulum system belongs to the class of under-actuated mechanical systems having fewer control inputs than degrees of freedom. This renders the control task more challenging making the inverted pendulum system a classical benchmark for testing different control techniques.

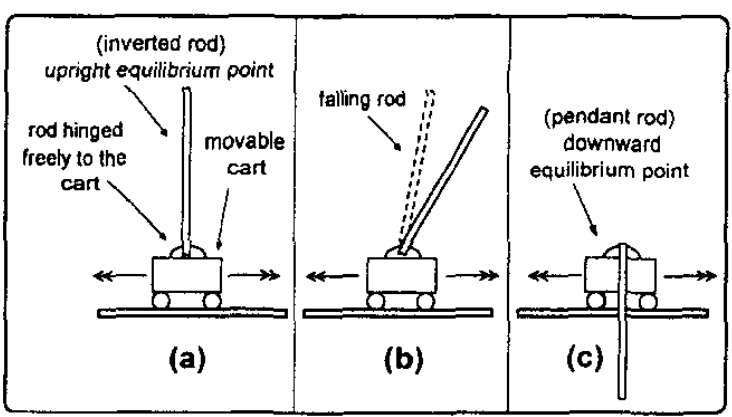

Fig. I.1. Equilibrium Points

There are a number of different versions of the inverted pendulum system offering a variety of interesting control challenges. The most common types are: the single rod on a cart inverted pendulum considered in [1]-[3], the Double Inverted Pendulum (DIP) on a cart setup [4], the rotational single-arm pendulum [5], [6] and the rotational two-link pendulum [7]. The control techniques involved are also numerous, ranging from simple conventional controllers to advanced control techniques based on modern non-linear control theory.

As a result of their nature, most of these papers deal with the problem in a purely theoretical manner without going into the practical considerations necessary in the design and implementation of a practical inverted pendulum rig. This paper aims at presenting a complete solution unifying the theoretical and practical aspects of the problem, along with a set of experimental results demonstrating the effectiveness of the complete implentented system. 
The paper is organized as follows: Section 2 presents a brief overview of the complete system. Section 3 deals with the mathematical dynamic model of the system used both for the computer simulations (MATLAB) and for the mathematical design of the controllers. Section 4 goes through the main steps in the design of the control algorithms their digital implementation and other practical issues. Section 5 presents some simulation and practical results, outlining any discrepancies between the two and finally several conclusions are drawn in Section 6.

\section{SYSTEM OVERVIEW}

This project consists in the design and implementation of both the physical system making up the inverted pendulum rig, and the control algorithms employed to attain the control task described in Section 1. The physical part of the system is made up of the mechanical setup composed of the motor driven cart and pendulum assembly, together with sensors and electronic circuitry. The control algorithms are implemented on a Digital Signal Processor (DSP) based system.

Figure 2.1 depicts the complete closed loop system. The DSP block is the main controller, made up of the TMS320C6711 DSP board from Texas Instruments equipped with the AED-103, an Input/Output (I/O) daughter module from Signalware Corporation. The system states $x$ and $\theta$, corresponding to the cart and pendulum displacements respectively, are obtained from incremental and absolute optical encoders mounted on the moving cart. These signals are fed to the DSP board which computes the other two states $\dot{\theta}$ and $\dot{x}$ (using finite backward difference equations), and uses the control algorithm to issue an output signal corresponding to a torque reference. This torque command is then fed as a reference input to the analogue ProportionalIntegral (PI) controller, regulating the motor torque accordingly by taking into account the motor dynamics. This controller uses the motor current, sensed by a Hall-effect sensor, as the feedback signal.

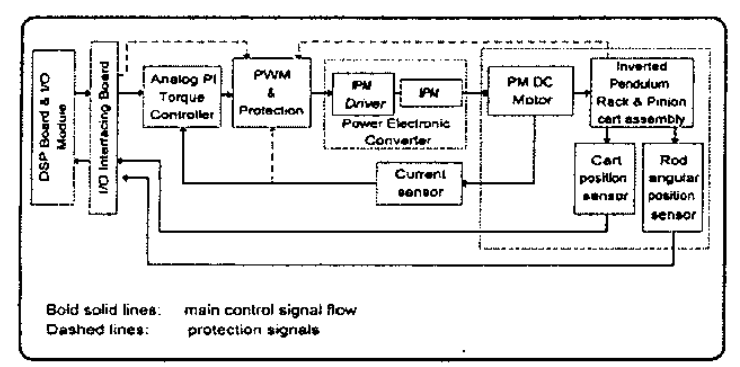

Fig. 2.1. Complete Closed Loop System

\section{Mathematical Modelling}

The dynamic model of the whole system consists of two separate sub-models, namely: the non-linear model of the inverted pendulum, and a linear model of the Permanent
Magnet (PM) DC motor powering the cart. This division was adopted in order to keep the non-linear dynamic equations of the inverted pendulum as simple as possible, which is imperative for the design of the non-linear swing-up controller.

The non-linear inverted pendulum model considers the force on the cart as the input, and the angle of the pendulum and cart displacement as the outputs. The motor model considers the motor terminal voltage as its input and the shaft torque as its output. Both models are derived separately and the resulting dynamic equations are then used in the design stage to develop two different control systems operating simultaneously at well distinct bandwidths (cascade control).

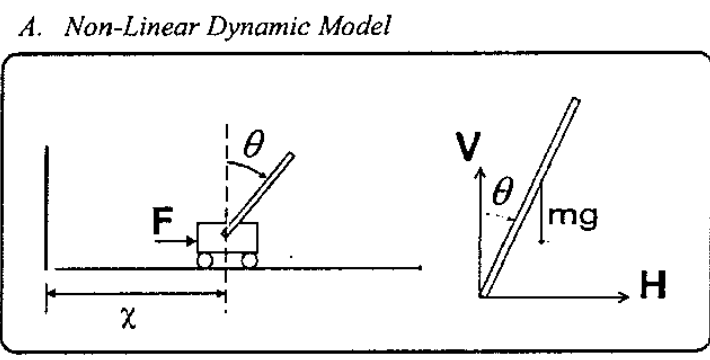

Fig. 3.1. Rod and cart setup and its force diagram

Referring to Figure 3.1 and applying Newton's $2^{\text {nd }}$ law at the centre of gravity of the pendulum along the horizontal \& vertical components yields

$$
\begin{aligned}
& V-m g=m \frac{d^{2}}{d t^{2}}(L \cos \theta), \\
& H=m \frac{d^{2}}{d t^{2}}(x+L \sin \theta) .
\end{aligned}
$$

Taking moments about the centre of gravity yields the torque equation

$I \ddot{\theta}+c \dot{\theta}=V L \sin \theta-H L \cos \theta$.

Applying Newton's $2^{\text {nd }}$ law for the cart yields

$F-H=M \ddot{x}+k \dot{x}$.

Where $m$ is the mass at the Centre Of Gravity (COG) of the pendulum; $M$ is the mass of the cart; $L$ is the distance from the COG of the pendulum to the pivot; $x$ is the horizontal displacement of the cart; $g$ is the gravitational acceleration; $\theta$ is the rod angular displacement; $k$ is the cart viscous friction coefficient; $c$ is the pendulum viscous friction coefficient; $I$ is the moment of inertia of the pendulum about the COG; $V \&$ $H$ are the vertical \& horizontal reaction forces on the rod and $F$ is the horizontal control force on the cart. Combining (1) to (4), the non-linear mathematical model of the cart and pendulum system is obtained and is given by (5) and (6). 


$$
\begin{aligned}
& \ddot{\theta}=\frac{1}{l+L^{2} m}[\operatorname{Lm}(g \sin \theta-\ddot{x} \cos \theta)-c \dot{\theta}] \\
& \ddot{x}=\frac{1}{M+m}\left[F-\operatorname{Lm}\left(\ddot{\theta} \cos \theta-\dot{\theta}^{2} \sin \theta\right)-k \dot{x}\right]
\end{aligned}
$$

\section{B. Linearized Model in State-Space Form}

Equations (5) and (6) were used to model the open-loop inverted pendulum (motor dynamics not included) during simulations. The same non-linear model was used for the design of the non-linear swing-up controller. However, for the design of the linear state-feedback controller, used for stabilization, a linearized version of these equations was used. The inverted position of the pendulum corresponds to the unstable equilibrium point $(\theta, \dot{\theta})=(0,0)$. This corresponds to the origin of the state space. In the neighbourhood of this equilibrium point, both $\theta$ and $\dot{\theta}$ are very small (in rad \& $\mathrm{rad} / \mathrm{sec}$ respectively). In general, for small angles of $\theta$ and $\theta$ : $\sin (\theta) \approx \theta, \quad \cos (\theta) \approx 1$ and $\left(\theta^{\prime}\right)^{2} \theta \approx 0$. Using these approximations in (5) and (6), the mathematical model linearized around the unstable equilibrium point of the inverted pendulum is obtained, and given by (7) and ( 8 )

$$
\begin{aligned}
\ddot{\theta} & =\frac{1}{I+L^{2} m}[\operatorname{Lm}(g \theta-\ddot{x})-c \dot{\theta}] \\
\ddot{x} & =\frac{1}{M+m}[F-L m \ddot{\theta}-k \dot{x}] .
\end{aligned}
$$

To get these two equations into valid state space linear form both $\ddot{x}$ and $\ddot{\theta}$ must be functions of lower order terms only. Hence, $\ddot{x}$ must be substituted for in (7) using (8), and similarly $\ddot{\theta}$ substituted for in (8) using (7). Writing the resulting equations in matrix form, the linearized state-space model is obtained and is given by the matrix linear equations (9) and (10).

$$
\begin{aligned}
& \dot{s}=\left[\begin{array}{lll}
0 & 1 & 0 \\
0 & -k v_{2} & \frac{-(L m)^{2} g v_{2}}{J+L^{2} m} \\
0 & 0 & 0 \\
0 & \frac{L m k v_{1}}{M+m} & L m g r_{1}
\end{array}\right. \\
& \underline{y}=\left[\begin{array}{llll}
1 & 0 & 0 & 0 \\
0 & 0 & 1 & 0
\end{array}\right] \underline{\underline{s}}
\end{aligned}
$$$$
\left.\begin{array}{l}
0 \\
\frac{L m c v_{2}}{1+L^{2} m} \\
-c v_{1}
\end{array}\right]=\left[\begin{array}{l}
0 \\
v_{2} \\
0 \\
\frac{-L m v_{1}}{M+m}
\end{array}\right] F
$$

Where state vector $\underline{s}=\left[\begin{array}{llll}x & \dot{x} & \theta & \theta^{\mathrm{\theta}}\end{array}\right]^{\mathrm{T}}$,

output vector $\underline{y}=\left[\begin{array}{ll}x & \theta\end{array}\right]^{\mathrm{T}}$,

$$
v_{1}=\frac{M+m}{I(M+m)+L^{2} m M} \text { and } v_{2}=\frac{I+L^{2} m}{I(M+m)+L^{2} m M} \text {. }
$$

\section{Permanent Magnet DC Motor Dynamics}

The mathematical model of the DC PM motor was required for the design of the motor torque PI controller. The mathematical model for the motor, considering the motor terminal voltage as input and the shaft torque as output, is given by the transfer function in (15).

$r(s) / V_{r}(s)=[(K, / L) s] /\left[s^{2}+(R / L) s+\left(K^{2} / L\right)\right]$

Where $L$ is the terminal inductance of the motor; $R$ is the terminal resistance; $K$, is the torque constant; $J$ is the rotor inertia; $V_{T}$ is the terminal voltage and $T$ is the developed torque.

\section{SYSTEM DESIGN}

The inverted pendulum control was split in two main phases: the swing-up phase and the stabilizing phase. The former uses a non-linear controller to swing-up the pendulum, keeping the cart within a limited travel range on the rail. The latter uses a linear state-feedback controller to stabilize the pendulum in the inverted position once it approaches the upper unstable equilibrium point. It is also required to home the cart to a reference point on the rail, once the pendulum is stabilized. A transition algorithm switches smoothly from one control phase to the other.

\section{A. Swing-Up Control}

The method proposed in [1] and adopted for the swing-up control phase in this project is briefly discussed in this section. Reference [1] contains a thorough explanation of this method. This technique aims at swinging up the pendulum, while keeping the cart within a limited horizontal travel on the rail. This is achieved by satisfying a particular mathematical condition, derived from the mechanical energy equations of the pendulum, while constructing a linear servo system, using a sinusoidal reference input generated from the pendulum trajectory. The total mechanical energy of the pendulum $V$, and its derivative $\dot{V}$, are given by (16) and (17).

$$
\begin{aligned}
& V=\frac{1}{2} m L^{2} \theta^{2}+m g L(1-\cos \theta) \\
& \dot{V}=m L \dot{\theta}(\cos \theta) \ddot{x}
\end{aligned}
$$

From (16) and (17), it is clear that $V$ can be increased or decreased by changing the sign (sgn) of $\ddot{x}$ in accordance with that of $\dot{\theta} \cos \theta$. If $\operatorname{sgn}(\ddot{x})=\operatorname{sgn}(\dot{\theta} \cos \theta)$ then $\dot{V}>0$, similarly if $\operatorname{sgn}(\ddot{x})=-\operatorname{sgn}(\dot{\theta} \cos \theta)$ then $\dot{V}<0$. Hence, energy can be pumped into the pendulum by generating $\ddot{x}$ (an acceleration on the cart) satisfying the sign conditions listed above. However, one cannot concentrate on swinging-up the pendulum only, without considering the finite cart travel (limited range for $x$ ). Therefore, $\ddot{x}$ has to be controlled whilst - keeping the constraint on $x$ in mind. Basically, the design method proposed in [1] suggests; constructing a control law such that the resulting closed loop system is linear (through feedback linearization) and of the form of a second order servo system for $x$, having a sinusoidal reference input to 
ensure the desired bounded nature of $x$. This reference input is derived from $(\theta, \dot{\theta})$, and generates $\ddot{x}$ satisfying the sign condition given above. This is done in order to control $V$ to the prescribed value corresponding to the energy of the pendulum at the upright equilibrium point. Since the pair of $(\theta, \dot{\theta})$ that makes $V$ equals to the desired value is not unique, the upright equilibrium point cannot be stabilized using only this control method. For this reason a different control law is utilized when the pendulum approaches the upright equilibrium point. This is referred to as stabilizing control.

\section{B. Stabilizing Control}

This control method is based on state-space poleplacement design techniques using the linearized model of the inverted pendulum. This implies that the stabilizing control by itself will only ensure local stability, in the vicinity of the upright equilibrium point, the point about which the equations were linearized. The pole-placement technique permits the design of a linear controller that achieves arbitrary desired closed loop poles. The desired poles should be chosen wisely such that some desired closed loop characteristics are achieved. The final control law, from this design, is the result of a matrix multiplication between the state vector $\underline{s}$ and a gain matrix of compatible dimensions $K^{T}$, such that $F=-K^{T} \underline{s} . \quad$ In this particular design, a small settling time and a high damping ratio were required. To meet these specifications, the closed loop poles were placed at $s=\mu_{i}(i=1,2,3,4)$, where $\mu_{1}=-4.05+j 1.962$, $\mu_{2}=-4.05-j 1.962, \mu_{3}=-10$ and $\mu_{4}=-10$. Basically, $\mu_{1}$ and $\mu_{2}$ are a pair of dominant closed-loop poles with damping factor $\xi=0.9$ and natural frequency $\omega_{n}=4.5 \mathrm{rad} / \mathrm{sec}$, resulting in a settling time of approximately 1 second. The other two poles are located far to the left of the dominant pair of closed loop poles and therefore, their effect over the overall response is minimal.

\section{Transition Algorithm}

An intermediate algorithm was designed to switch from the swing-up controller to the stabilizing controller and viceversa, depending on the state variables $\theta$ and $\dot{\theta}$. Actually, this algorithm performs a smooth transition from one control law to the other by averaging the outputs of the two controllers in the transition region. This avoids what is known as hard switching, which may upset the system due to parameter uncertainties and un-modelled dynamics. Basically, if $\theta$ and $\theta$ are both close to zero, only the stabilizing controller is used. Similarly if they are much higher than zero, only the swing-up algorithm is used. In between these two extremes a region was created, the transition region, in which both algorithms are processed and weighted accordingly, leading to soft switching.

\section{Digital Implementation}

The swing-up and stabilizing controllers were implemented on the TMS320C6711 DSP. For this reason, the corresponding control laws had to be implemented in software. For linear control systems, the effects of sampling are usually taken into consideration by discretization; a conversion of the continuous time dynamic equations, taking into account the sampling process and the holding devices. However, non-linear physical systems that are continuous in nature are hard to meaningfully discretize [8], since wellknown discretization techniques, like the Z-transform, do not apply for non-linear systems. Therefore, non-linear digital control systems are usually treated as continuous time systems in analysis and design. This approach is only' justified if high sampling rates, compared to the bandwidth of the plant under control, are used. Another important factor in digital control systems is that the time period between the sensing of information and actuation should be much shorter than the sampling period itself. In this system a sampling frequency of $100 \mathrm{~Hz}$ was chosen after mathematical analysis revealed that the highest bandwidth in the system is around $1.8 \mathrm{~Hz}$. These requirements and the computational complexity of the non-linear control algorithm were the main reasons for using a floating point DSP to implement the digital controller.

\section{E. Physical Setup}

The rig consists of a two-meter L-shaped wooden bench equipped with a linear-guide rail on its horizontal face and a toothed rack mounted on the vertical face. The cart slides over the linear-guide rail using a compatible linear-guide carriage fitted at the bottom The cart is driven by a $250 \mathrm{~W}$, $36 \mathrm{~V}$ Rare-Earth (RE) PM DC motor, driven through a 4 quadrant full-bridge converter using a bipolar voltage switching technique. An incremental encoder and an absolute encoder provided the two system states, $x$ and $\theta$ respectively. The motor and the incremental encoder are equipped with steel pinions to engage firmly in the toothed rack. An analogue electronic Proportional Integral (PI) torque controller was designed to improve the overall system performance by creating a fast inner torque control loop cascaded to the main control loop as shown in Figure 2.1.

Finally, the DSP system interfaced to the rest of the system through a signal conditioning board, purposely designed to help in reducing the noise levels on the signals.

\section{SIMULATION \& PRACTICAL RESUltS}

The controllers discussed in Section 4, were simulated using the non-linear model of the pendulum. The inner loop dynamics were neglected in these simulations. This is valid since in practice the inner control loop was designed to have - a much higher bandwidth than the pendulum dynamics. 


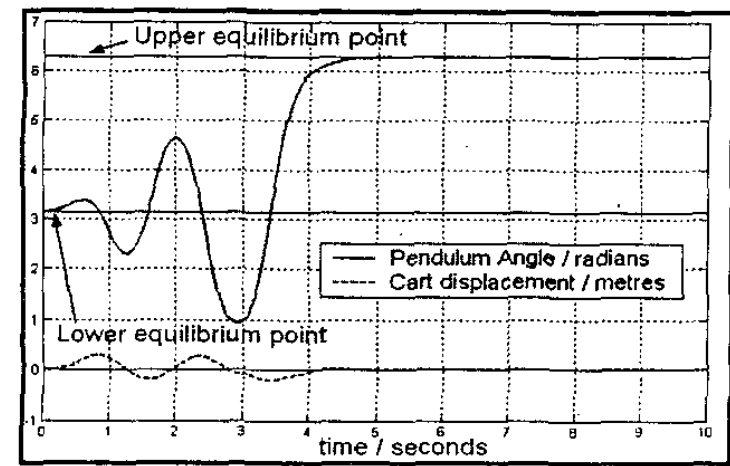

Fig. 5.1. Simulation of Swing-Up \& Stabilization

Figure 5.1 shows the simulation plots for $\theta$ and $x$ during swing-up and stabilization. Initially the pendulum is in the pendant position. It swings-up gradually, responding to the bounded oscillations of the cart. Up to 3.91 seconds the swing-up controller is in control. Then, the transition algorithm takes over till 3.99 seconds. The state-feedback controller takes over completely for the rest of the time, stabilizing the pendulum in the inverted position and homing the cart to the reference point on the rail. Note that the pendulum swings-up and stabilizes in less than 5 seconds.

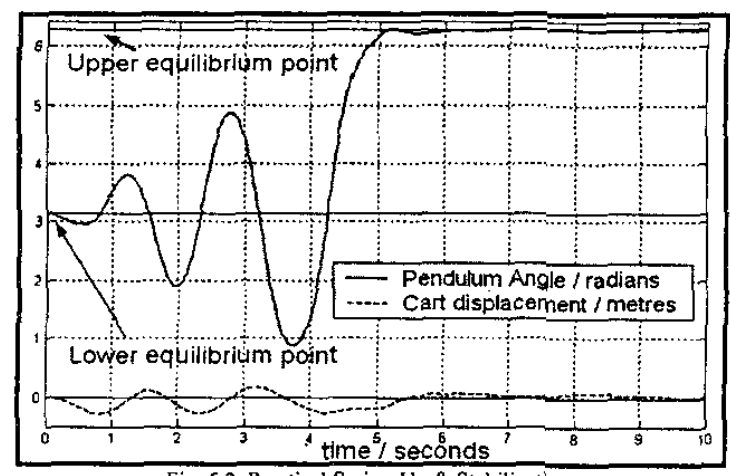

Fig. 5.2. Practical Swing-Up \& Stabilization

Figure 5.2 shows the actual experimental plots for $\theta$ and $x$ acquired from a swing-up and stabilization test using the physical inverted pendulum system designed and implemented in this project. It is clear that the pendulum swung-up from its initial pendant position and stabilized in the inverted position, with the cart homing back to its initial reference point, in less than 5 seconds. The cart oscillations are also limited between $6.29 \mathrm{~cm}$ and $28.6 \mathrm{~cm}$ during swingup, showing the effectiveness of the servo cart dynamics imposed by feedback linearization as proposed in [1]. These results are almost a replica of the simulations, with the slight difference that in practice both the cart and the pendulum keep oscillating slightly about the reference after they are stabilized. These oscillations are less than $5 \mathrm{~cm}$ for the cart and $1^{\circ}$ for the pendulum and are accounted for by the continuous air disturbances on the rod, non-linear un- modelled dynamics, such as Coulomb friction, pinion backlash, motor dead-zone and magnetic hysteresis, and other mechanical imperfections.

\section{CONCLUSIONS}

The results presented in Section 5 verify that the system designed and implemented in this project was successful. The control task stated in Section 1 was completely fulfilled, i.e, the pendulum swung-up from its natural pendant position according to the algorithm developed in [1], and stabilized in the inverted open-loop unstable position using state-feedback pole-placement. The cart also homed back quickly to a reference position on the rail. The controlled inverted pendulum was proven to be highly robust for external rod disturbances and the controller exhibited stability for both $x$ and $\theta$ given any initial conditions. Short video clips of the operating inverted pendulum can be found at [9], and further technical details about this project can be found at [10].

\section{ACKNOWLEDGMENT}

The author would like to thank his project supervisor $\mathrm{Dr}$. Ing. Simon G. Fabri B.Elec.Eng.(Hons.), MSc (Eng), PhD, for his supervision, assistance and support. Further acknowledgments go to the University of Malta for the financial support.

\section{REFERENCES}

[1] K. Yoshida, "Swing-up control of an inverted pendulum by energybased methods," proceedings of the American control conference, pp. 4045-4047, 1999.

[2] K.J. Astrom and K. Furuta, "Swinging up a pendulum by energy control," presented at $13^{\text {th }}$ IFAC world congress, 1996.

[3] A. Ohsumi and T. Izumikawa, "Norlinear control of swing-up and stabilization of an inverted pendulum," proceedings of the $34^{\text {th }}$ conference on decision \& control, pp. 3873-3880, 1995.

[4] W. Zhong and H. Rock, "Energy and passivity based control of the double inverted pendulum on a cart," presented at IEEE Conference on Control Applications, 2001.

[5] K. Furuta and M. Yamakita, "Swing up control of inverted pendulum," IECON '91, pp. 2193-2198, 1991.

[6] K. Funuta and M. Yamakita, "Swing-up control of inverted pendulum using pseudo-state feedback," proceedings of Instn. Mech Engrs, vol. 206, pp. 263-269, 1992.

[7] M. Yamakita and K. Furuta, "Toward robust state transfer control of TITech double pendulum," The Astrom symposium on control, B. Wittenmark and $A$. Rantzer.

[8] J.J.E. Slotine and W. Li, Applied Non-Linear Control, Prentice- Hall Inc., New Jersey, 1991.

[9] M. Bugeja, "Non-linear swing-up and stabilizing control of an inverted pendulum system," http://www.eng.um.edu.mt/ sgfabr/bugeja,html.

[10] M. Bugeja, "Non-linear swing-up and stabilizing control of an inverted pendulum system," B.Eng.(Hons.) thesis, University of Malta, Msida, Malta, May 2002. 